DRAFT VERSION SEPTEMBER 18, 2018

Preprint typeset using LTEX style emulateapj v. 03/07/07

\title{
PERIODIC RADIO EMISSION FROM THE M7 DWARF 2MASS J13142039+1320011: IMPLICATIONS FOR THE MAGNETIC
} FIELD TOPOLOGY

\author{
M. MCLEAN ${ }^{1}$, E. BERGER ${ }^{1}$, J. IRWIN $^{1}$, J. FORBRICH $^{1}$, AND A. REINERS ${ }^{2}$ \\ Draft version September 18, 2018
}

\begin{abstract}
We present multi-epoch radio and optical observations of the M7 dwarf 2MASS J13142039+1320011. We detect a $\sim 1$ mJy source at $1.43,4.86,8.46$ and $22.5 \mathrm{GHz}$, making it the most luminous radio emission over the widest frequency range detected from an ultracool dwarf to date. A $10 \mathrm{hr}$ VLA observation reveals that the radio emission varies sinusoidally with a period of $3.89 \pm 0.05 \mathrm{hr}$, and an amplitude of $\approx 30 \%$ at $4.86 \mathrm{GHz}$ and $\approx 20 \%$ at $8.46 \mathrm{GHz}$. The periodicity is also seen in circular polarization, where at $4.86 \mathrm{GHz}$ the polarization reverses helicity from left- to right-handed in phase with the total intensity. An archival detection in the FIRST survey indicates that the radio emission has been stable for at least a decade. We also detect periodic photometric variability in several optical filters with a period of $3.79 \mathrm{hr}$, and measure a rotation velocity of $v \sin i=45 \pm 5$ $\mathrm{km} \mathrm{s}^{-1}$, in good agreement with the radio and optical periods. The period and rotation velocity allow us to place a lower limit on the radius of the source of $\gtrsim 0.12 \mathrm{R}_{\odot}$, about $30 \%$ larger than theoretical expectations. The properties of the radio emission can be explained with a simple model of a magnetic dipole mis-aligned relative to the stellar rotation axis, with the sinusoidal variations and helicity reversal due to the rotation of the magnetic poles relative to our line of sight. The long-term stability of the radio emission indicates that the magnetic field (and hence the dynamo) is stable on a much longer timescale than the convective turn-over time of $\sim 0.2 \mathrm{yr}$. If the radio emission is due to the electron cyclotron maser process, the inferred magnetic field strength reaches at least $8 \mathrm{kG}$.
\end{abstract}

Subject headings: radio continuum:stars — stars:activity — stars:low-mass, brown dwarfs — stars:magnetic fields

\section{INTRODUCTION}

An unexpected result from radio observations of low mass stars and brown dwarfs was the recent discovery of periodic radio emission tied to the stellar rotation (Berger et al. 2005; Hallinan et al. 2006, 2007; Berger et al. 2009). Previously thought to be incapable of generating and dissipating strong and stable magnetic fields, observations in recent years indicate that at least $10 \%$ of fully convective late-M and L dwarfs possess stable and large-scale magnetic fields (Berger 2002, 2006; Berger et al. 2010). When combined with X-ray and $\mathrm{H} \alpha$ observations (Mohanty \& Basri 2003; Reiners \& Basri 2008; Berger et al. 2010), Zeeman broadening of $\mathrm{FeH}$ molecular lines in Stokes $I$ (Reiners \& Basri 2007), and spectropolarimetry in Stokes $V$ (Zeeman Doppler Imaging: ZDI; Donati et al. 2006), a picture of the magnetic topology of fully convective ultracool dwarfs is now beginning to emerge.

Zeeman broadening observations provide a measure the average surface magnetic field, $B f$ (where $B$ is the magnetic field and $f$ is the covering fraction) and have been used to study moderately rotating objects as cool as M9 (Reiners \& Basri 2010). These observations uncovered average magnetic field strengths of up to a few $\mathrm{kG}$. The ZDI technique has been used to study the large scale structure and topology of magnetic fields (as it is sensitive only to the net polarization in Stokes $V$ ) in objects down to spectral type M8. At the fully convective boundary (spectral type of $\sim$ M3) these studies hint at a transition from nonaxisymmetric toroidal fields to predominantly poloidal, ax-

\footnotetext{
${ }^{1}$ Harvard-Smithsonian Center for Astrophysics, 60 Garden Street, Cambridge,MA 02138

${ }^{2}$ Universität Göttingen, Institut für Astrophysik, Friedrich-Hund-Platz 1, 37077 Göttingen, Germany
}

isymmetric field topologies (Donati et al. 2008; Morin et al. 2008), although the systematic uncertainties and general detectability of field topologies across a wide range in stellar parameters are not fully understood. Similarly, there seems to be an increase in the ratio of magnetic fluxes measured in circularly polarized to unpolarized spectral lines at this boundary, apparently confirming that the fully convective objects have increasingly large-scale, low-multipole field topologies. However, a number of fully convective mid-M dwarfs, potentially belonging to a younger population, still appear to possess small-scale complex fields (Morin et al. 2010), although recent observations seem to question this idea (Reiners 2010).

In the context of magnetic activity, the correlation between X-ray and radio emission, which holds across several orders of magnitude in each quantity and over a wide spectral type range (Guedel \& Benz 1993; Benz \& Guedel 1994), sharply breaks down around spectral type M7-M8 (Berger et al. 2001; Berger 2002; Berger et al. 2010). X-ray and $\mathrm{H} \alpha$ activity also decline precipitously around the same spectral type (Mohanty \& Basri 2003; Reiners \& Basri 2008; Berger et al. 2010). Finally, the $\mathrm{H} \alpha$ and X-ray rotationactivity relations also break down near the $\mathrm{M} / \mathrm{L}$ transition (Mohanty \& Basri 2003; Berger et al. 2008; Reiners \& Basri 2010). The origin of these changes remains unclear, in particular whether they are related to a change in the magnetic dynamo itself, or to a change in the way magnetic energy is released in the cool and largely neutral atmospheres.

Radio activity in ultracool dwarfs, on the other hand, appears to be equally strong (or stronger when normalized to the bolometric luminosity) than in early-M dwarfs (Berger 2002, 2006; Berger et al. 2010). Among the active population of ultracool dwarfs, the handful of objects with periodic radio emission exhibit diverse behavior. In particular, the wide variation in the temporal behavior, emission bandwidth, and 
fractional polarization suggest that there are a number of different processes at work. Three objects (TVLM 513-46546: Hallinan et al. 2006; LSR 1835+3259: Hallinan et al. 2007; and 2M 0746+2000: Berger et al. 2009) have been observed to emit short-duration, highly polarized pulses with apparently narrow bandwidth. On the other hand, 2M 0036+1821 exhibits periodic variability with a different structure, rising and falling in a roughly sinusoidal manner (Berger et al. 2005). In all four objects, the observed periods, which range from two to three hours, are in close agreement with the known rotation velocities $(v \sin i)$.

Two primary emission mechanisms have been proposed to explain the observed radio emission: gyrosynchrotron radiation and the electron cyclotron maser (ECM) instability (Berger 2006; Treumann 2006; Hallinan et al. 2008; Berger et al. 2009). The short-duration periodic pulses have been attributed to a coherent process such as ECM due their small bandwidth and high degree of circular polarization (Hallinan et al. 2007; Berger et al.2009). However, the mechanism for the sinusoidal radio emission from $2 \mathrm{M} \mathrm{0036+1821,}$ as well as the quiescent emission from several ultracool dwarfs, is still under debate, partly because of the low levels of circular polarization and the much wider bandwidth.

Here we present multi-wavelength observations of a newly discovered radio-emitting M7 dwarf, 2MASS J13142039+1320011 (hereafter, 2M 1314+1320), which exhibits unique emission properties among the population of radio active ultracool dwarfs. It has the brightest radio emission over the widest frequency range of any ultracool dwarf to date (detected from 1.4 to $22.5 \mathrm{GHz}$ with a nearly flat spectrum), and presents the first clear example of sinusoidal emission (in phase at 4.96 and $8.46 \mathrm{GHz}$ ). The periodicity is also detected in circular polarization, including a helicity reversal that provides insight into the topology of the emission regions. Photometric observations reveal periodic variability with the same period as in the radio band, indicative of a large-scale magnetic spot structure. The unique radio properties of $2 \mathrm{M} 1314+1320$, and its location at the cusp of the transition in magnetic field properties (spectral type M7), make it an invaluable laboratory for fully convective dynamos.

\section{OBSERVATIONS}

\subsection{Target Properties}

We identified the M7 ultracool dwarf 2M 1314+1320 as a radio-emitting source in 2007 during a large Very Large Array $\left(\mathrm{VLA}^{3}\right)$ survey of nearby mid- and late-M dwarfs (McLean \& Berger 2011). It is a high proper motion object $\left(\delta \mathrm{RA} \approx-244\right.$ mas $\mathrm{yr}^{-1}, \delta \mathrm{Dec} \approx-186$ mas $\mathrm{yr}^{-1}$; Lépine \& Shara 2005), consistent with the young disk population, and has a parallactic distance of 16.4 pc (Lépine et al. 2009). Observations in the $i$ and $z$ bands with the Lucky Imaging technique have demonstrated that it is a resolved binary with a separation of $0.1^{\prime \prime}$ (about $1.6 \mathrm{AU}$ ), and a companion that is fainter by about 1 mag (Law et al. 2006). $2 \mathrm{M} \mathrm{1314+1320}$ has strong $\mathrm{H} \alpha$ emission, with an equivalent width of $-54 \AA$, corresponding to $L_{\mathrm{H} \alpha} / L_{\text {bol }} \approx-3.2$ (Lépine et al. 2009), consistent with the rotationally-saturated level observed in early- and mid-M dwarfs.

\footnotetext{
3 The VLA is operated by the National Radio Astronomy Observatory, a facility of the National Science Foundation operated under cooperative agreement by Associated Universities, Inc.
}

\subsection{VLA Observations}

We observed 2M 1314+1320 with the VLA on several occasions and at several frequencies (1.43, 4.86, 8.46 and 22.5 $\mathrm{GHz})$. Data were acquired as part of programs AB1245 and AB1312 (PI: Berger). The details of the observations are provided in Table 1. All observations were obtained in the standard continuum mode with $2 \times 50 \mathrm{MHz}$ contiguous bands. During the $10 \mathrm{hr}$ simultaneous observation we used fourteen antennas at $4.86 \mathrm{GHz}$ and twelve antennas at $8.46 \mathrm{GHz}$. Phase calibration was performed using $\mathrm{J} 1309+119$, while the flux density scale was calibrated using 3C $286(\mathrm{~J} 1331+305)$. We reduced and analyzed the data using the Astronomical Image Processing System (AIPS). We inspected the visibility data for quality, and noisy points were removed. In each of the observations a source coincident with the position of

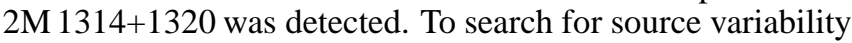
in the 10-hr simultaneous observation, we constructed light curves by plotting the real part of the complex visibilities at the position of $2 \mathrm{M} 1314+1320$ as a function of time using the AIPS DFTPL routine.

2M 1314+1320 is also identified in data from the Faint Images of the Radio Sky at Twenty-Centimeters (FIRST) survey, where it was observed in December 1999 at $1.43 \mathrm{GHz}$ with a flux density of $1126 \pm 123 \mu \mathrm{Jy}$. A comparison of the position in the FIRST data and in our subsequent VLA observations

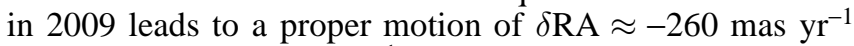

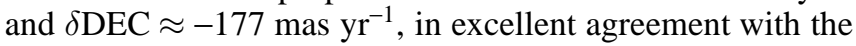
known proper motion of 2M 1314+1320.

\subsection{VLBI Observations}

We observed 2M1314+1320 with the 10-antenna Very Long Baseline Array (VLBA) and the Green Bank Telescope (GBT) at $8.46 \mathrm{GHz}$ on 2010 April $12 \mathrm{UT}$ for approximately $8 \mathrm{hr}$ (program BM0327; PI: McLean). Phase-referenced observations were carried out with a data rate of $512 \mathrm{Mbit} \mathrm{s}^{-1}$ in dual polarization, using two-bit sampling. Eight base-band channels of $8 \mathrm{MHz}$ bandwidth each were used, spread over at total base-width of $32 \mathrm{MHz}$. The correlator dump time was $1 \mathrm{~s}$. The complex gain calibrator was $\mathrm{J} 1309+1154$, located at a distance of $1.8^{\circ}$ from the target. There is a gap of about $50 \mathrm{~min}$ in the resulting light curve of $2 \mathrm{M} 1314+1320$ due to geodetic quasar observations intended to improve the astrometry.

We detected 2M 1314+1320 with a flux density of $F_{\nu}=$ $757 \pm 53 \mu \mathrm{Jy}$ at $8.46 \mathrm{GHz}$ with $14 \pm 4 \%$ circular polarization. The source is unresolved with a beam size of $2 \times 1$ mas, corresponding to an upper limit on the source diameter of about 25 stellar radii (Figure 11). This is about an order of magnitude larger than the expected scale of the magnetic field. The flux density is lower by about $27 \%$ from the average of the VLA observations, most likely due to a loss of flux caused by phase calibration errors. We tested this hypothesis by eliminating the longest baselines (i.e., reducing the angular resolution) and recovered the same flux density. In addition, we searched for potential emission from the secondary star in the system at a level corresponding to the missing flux $(\approx 0.3$ mJy) but we did not detect any other source above a $4 \sigma$ level of about $0.2 \mathrm{mJy}$ within a $0.8^{\prime \prime} \times 0.8^{\prime \prime}$ region centered on the detected source.

\subsection{Optical Spectroscopy}

To measure the projected rotation velocity, $v \sin i$, we obtained a high-resolution spectrum with the Magellan Inamori 
Kyocera Echelle (MIKE) spectrograph mounted on the Magellan/Clay 6.5-m telescope on 2009 May 19 UT. The single $1000 \mathrm{~s}$ spectrum was reduced using a custom reduction pipeline $^{4}$ written in Python. Wavelength calibration was performed using ThAr arc lamps, and air-to-vacuum and heliocentric corrections were applied. We measured the rotation velocity by cross-correlating the spectrum with a template spectrum of the inactive star G1 876 (Reiners \& Basri 2008). The value of $v \sin i$ was determined by comparing the width of the correlation function to cross-correlations between the template and artificially broadened versions of the template spectrum (see for example Reiners \& Basri 2006). We derive $v$ sini from several spectral orders and find $45 \pm 5 \mathrm{~km} \mathrm{~s}^{-1}$. There is no evidence for a spectroscopic binary. $\mathrm{H} \alpha$ emission was detected with an equivalent width of $9.9 \AA$, corresponding to $L_{\mathrm{H} \alpha} / L_{\mathrm{bol}} \approx-4.0$, lower than previous measurements (Lépine et al. 2009).

We also observed 2M 1314+1320 using the Low Dispersion Survey Spectrograph (LDSS3) on Magellan/Clay 6.5-m telescope on 2009 February 25 UT. A total of six $300 \mathrm{~s}$ integrations were obtained with the VPH-Blue grism using a $1^{\prime \prime}$ slit. The data were reduced using standard procedures in IRAF, and the wavelength calibration was performed using $\mathrm{HeNeAr}$ arc lamps. We detect non-variable $\mathrm{H} \alpha$ emission in all the individual spectra with an average equivalent width of $\approx 14.6$ $\AA$, corresponding to $L_{\mathrm{H} \alpha} / L_{\mathrm{bol}} \approx-3.8$.

\subsection{Photometric Monitoring}

To study the photometric behavior of $2 \mathrm{M} 1314+1320$ we observed the source on two consecutive nights (2010 April 6 and 7 UT) using KeplerCam on the Fred Lawrence Whipple Observatory (FLWO) 1.2-m telescope for about $7 \mathrm{hr}$ on each night. The observations alternated between the $g$ - and $i$-band filters with individual exposures of 180 and $60 \mathrm{~s}$, respectively. The data were reduced using standard procedures in IRAF. Relative photometry was performed using the Difference Imaging Photometry Pipeline phot pipe (Rest et al. 2005).

Longer term photometric monitoring (2009 January 11 to 2010 March 18 UT) was performed with a single telescope of the MEarth array at the Fred Lawrence Whipple Observatory. MEarth consists of 8 identical $0.4-\mathrm{m}$ robotic telescopes, and is dedicated to a search for super-Earth exoplanets in the habitable zones of nearby mid- to late-M dwarfs (Nutzman \& Charbonneau 2008). All observations were taken with a $715 \mathrm{~nm}$ long-pass filter, with the red end of the bandpass defined by the CCD quantum efficiency curve. Exposure times for data taken between 2009 January 11 and June 23 UT were $28 \mathrm{~s}$ with the telescope defocused to 6 pixels FWHM; data between 2009 December 5 and 2010 March 18 UT were obtained with an exposure time of $11 \mathrm{~s}$ with the telescope operated in focus. Observations were scheduled at the default 20 min cadence until 2009 June 12 UT when the cadence was increased to $10 \mathrm{~min}$. On 2010 February 14 UT, we observed for the entire night at the highest possible cadence $(\approx 30 \mathrm{~s}$, including overheads).

Basic reductions and light curve generation were performed using an automated pipeline, based on the Monitor project pipeline (Irwin et al. 2007). A number of instrument-specific refinements have been made, and these will be described in full in a forthcoming publication (Berta et al. 2011, in prep).

4 http://www.ociw.edu/Code/mike
MEarth data have two known systematic effects remaining after the standard pipeline differential photometry corrections, which are of particular importance for the detection of continuous photometric modulations such as rotation, and are detailed in Irwin et al. (2011). These are the "meridian offset" and "common mode" effects. We follow the same method here to correct for these effects, fitting for them simultaneously with the source modulations.

\section{PROPERTIES OF THE RADIO EMISSION}

The two initial 1-hr observations of 2M 1314+1320 at 8.46 $\mathrm{GHz}$ demonstrate a stable flux density level of about 1150 $\mu \mathrm{Jy}$ and stable circular polarization of about $20 \%$. Compared to the FIRST detection in 1999 (at $1.43 \mathrm{GHz}$ ), the flux density level appears to be stable for a period of about $10 \mathrm{yr}$. The 10$\mathrm{hr}$ simultaneous observation obtained about $1.5 \mathrm{yr}$ later allows us to study the short-term temporal variability of the radio emission. The light curves are shown in Figure 2, The average flux density is $1099 \pm 18 \mu \mathrm{Jy}$ at $4.86 \mathrm{GHz}$ and $1032 \pm 16$ $\mu \mathrm{Jy}$ at $8.46 \mathrm{GHz}$, again indicative of stable long-term radio emission, as well as a flat spectrum. The corresponding average luminosities are $L_{\nu}(4.86)=(3.31 \pm 0.05) \times 10^{14}$ and $L_{\nu}(8.46)=(3.52 \pm 0.06) \times 10^{14} \mathrm{erg} \mathrm{cm}^{-2} \mathrm{~s}^{-1} \mathrm{~Hz}^{-1}$. Assuming that the emission comes from the primary object, the ratio relative to the bolometric luminosity is $\log \left(\nu L_{\nu} / L_{\mathrm{bol}}\right) \approx-5.9$. This value is about 5 times larger than the brightest radioemitting $\mathrm{M}$ dwarfs detected to date, and roughly comparable with the L3.5 dwarf 2M 0036+1821 (Berger et al. 2005).

The key feature of the $10 \mathrm{hr}$ observation is the clear periodic variation in the flux density with $P \approx 4 \mathrm{hr}$. Unlike the periodic radio emission detected in previous ultracool dwarfs (TVLM 513-46546, LSR 1835+32, and 2M 0746+2000; Hallinan et al. 2006, 2007; Berger et al. 2009), the variation in flux density is smooth and sinusoidal rather than consisting of short-duration pulses. A LombScargle periodogram of the data is shown in Figure 3 revealing a single significant peak at both frequencies with $P=3.92 \pm 0.06 \mathrm{hr}(4.86 \mathrm{GHz})$ and $3.85 \pm 0.08 \mathrm{hr}(8.46 \mathrm{GHz})$. This is consistent with a single period of $3.89 \mathrm{hr}$ at both frequencies. Cross-correlation of the two light curves shows that there is no detectable phase shift between the two frequencies, with a $3 \sigma$ upper limit of $\approx 3 \mathrm{~min}$, or about $0.01 P$ (Figure 3). We also fit the light curves with a sine-function using a leastsquares algorithm, and find $P=4.0 \pm 0.1 \mathrm{hr}(4.86 \mathrm{GHz})$ and $3.9 \pm 0.1 \mathrm{hr}(8.46 \mathrm{GHz})$. The amplitude of the variation at $4.86 \mathrm{GHz}$ is $32 \pm 4 \%$ and at $8.46 \mathrm{GHz}$ it is $22 \pm 3 \%$. We do not detect obvious periodicity during the $8 \mathrm{hr}$ VLBA observation, but note that the noise level in the light curve precludes the detection of variability at the level $20 \%$ as observed in the VLA observations.

The periodic variability is also detected in circular polarization. At $4.86 \mathrm{GHz}$, the average polarization during the $10 \mathrm{hr}$ observation is close to zero but it varies at a level of $24 \pm 10 \%$ of the total flux density, alternating between rightand left-handed circular polarization over a best fit period of $3.81 \pm 0.09 \mathrm{hr}$ from a Lomb-Scargle periodogram analysis (Figure 4). The periodicity of the circular polarization is correlated with the modulation of the total intensity, with the left-handed polarization coinciding with the peaks in total intensity. At $8.46 \mathrm{GHz}$ the average polarization is weaker, $\approx 10 \%$, with no significant periodicity, although the helicity of polarization may reverse during the peaks of the total intensity (Figure 4).

The radio spectral energy distribution (SED) between 1.43 
and $22.5 \mathrm{GHz}$ is shown in Figure 5, including all of our VLA observations and the FIRST survey data. Unfortunately, only short observations were made at 1.4 and $22.5 \mathrm{GHz}$, so we have no information about periodicity at these frequencies. If the flux density variations have a similar amplitude to that observed at 4.86 and $8.46 \mathrm{GHz}$, the measured flux may be biased low or high by about $20-30 \%$ depending on the phase of the light curve probed by these short observations. The broadband spectrum peaks at about $5 \mathrm{GHz}$, with a flat spectrum at higher frequencies, $\beta \approx-0.2\left(F_{\nu} \propto \nu^{\beta}\right)$. For comparison, in Figure 6 we show the multi-frequency data for TVLM 51346546 (M8.5) from 1.4 to $8.5 \mathrm{GHz}$ (Osten et al. 2006) and DENIS 1048-3956 (M8) from 4.9 to $24 \mathrm{GHz}$ (Ravi et al. 2011). The broad-band SED of $2 \mathrm{M} 1314+1320$ is significantly flatter than that of DENIS 1048-3956.

The SED of circularly polarized flux is shown in Figure 7 including the full range from the $10 \mathrm{hr}$ observation. It is unclear if the measurement from the single $1 \mathrm{hr}$ observation at $1.43 \mathrm{GHz}$ represents the true mean polarization or if the object oscillates between right- to left-handed polarization at this frequency as it does at $4.86 \mathrm{GHz}$. Interestingly, the polarization is consistently positive at $8.46 \mathrm{GHz}$. However, the $10 \mathrm{hr}$ observation has the lowest average polarization, indicating a mixture of negative circular polarization related to the overall flux variability. The level of circular polarization in the VLBA detection $(+14 \%)$ is consistent with the VLA observations.

The radio emission properties can be used to extract information on the magnetic field strength in the context of the expected emission mechanisms. The overall low degree of circular polarization, and the wide frequency range over which the radio emission is detected may be indicative of gyrosynchrotron radiation. However, the nearly flat spectrum corresponds to an electron power law distribution with an energy index of $p \approx 1.3$, which is unusually low compared to typical values of $p \sim 3-4$ (Güdel 2002). The magnetic field strength in the case of gyrosynchrotron emission can be estimated using the relation $f_{c} \approx 2.85 \times 10^{3} B^{0.5} \nu_{p}^{-0.5}$, indicating $B \approx 50 \mathrm{G}$ for a peak frequency of $\nu_{p} \approx 5 \mathrm{GHz}$ and a fraction of circular polarization of $f_{c} \approx 0.25$. On the other hand, electron cyclotron maser emission, which is produced primarily at the fundamental electron cyclotron frequency, $\nu_{c} \approx 2.8 \times 10^{6} \mathrm{~B}$ $\mathrm{Hz}$, require the magnetic field strength to vary from about 0.5 to $8 \mathrm{kG}$ within the emission region of $2 \mathrm{M} 1314+1320$ to explain the broad-band SED. We note that the flat spectrum is indicative of an even wider distribution of magnetic field strengths. The maximal value is much larger than the average surface magnetic field strengths measured on similar objects using Zeeman measurements $(1-4 \mathrm{kG}$; Reiners \& Basri 2007), but it is possible that the Zeeman technique is not sensitive to such fields if their spatial scale is relatively small.

\section{PROPERTIES OF THE OPTICAL EMISSION}

The $g$ - and $i$-band light curves from the FWLO 1.2-m observation are shown in Figure 8 The data exhibit the same periodicity seen in the radio, and the resulting phased light curves (with $P \approx 3.78 \mathrm{hr}$ ) are shown in Figure 9 The variability amplitude is $\approx 80 \mathrm{mmag}$ in $g$-band and $\approx 50 \mathrm{mmag}$ in $i$-band, and both bands are in phase. A phased light curve from the MEarth data is shown in Figure 10, with a period of $3.785 \mathrm{hr}$ determined from a Lomb-Scargle periodogram analysis (Figure 11). The variation amplitude in the $715 \mathrm{~nm}$ long-pass filter is $\approx 15$ mmag indicating an overall trend of decreasing variability at longer wavelengths.

The phased light curve is asymmetric, with a longer rise in brightness compared to the subsequent decline (the rise time from trough to peak is about $2.3 \mathrm{hr}$, while the decline back to minimum takes about $1.5 \mathrm{hr}$ ). The optical period is slightly shorter than the period determined from the VLA observations, by $6 \pm 3 \mathrm{~min}$. This subtle difference may be potentially due to differential rotation if the radio and optical variability arise at different latitudes on 2M 1314+1320. The MEarth and VLA observations could not be phased due to the large gap in the observations. The overall sinusoidal variability of $2 \mathrm{M} 1314+1320$ points to a large covering fraction for the $\operatorname{spot}(\mathrm{s})$, while the asymmetry is indicative of multiple or irregularly-shaped spots.

Photometric rotational modulation is common in M dwarfs and is generally attributed to magnetic spots. However, the cool neutral atmospheres of ultracool are expected to also form clouds that may produce periodic variability (Bailer-Jones 2002). Since 2M 1314+1320 is an M7 dwarf, its atmosphere is still fairly warm and dust clouds are less likely. The ratio of the variability amplitude in the $g$ and $i$ bands is about 1.6, which is consistent with a cool spot (Rockenfeller et al. 2006). For comparison, the radio active M8.5 dwarf TVLM 513-46546 exhibits photometric modulation with the same period as the radio variability, but the $g$ and $i$-band variations are anti-correlated, indicative of clouds rather than magnetic spots (Littlefair et al. 2008). Here the $g$ and $i$-band variations are in phase, indicating that magnetic spots are the likely explanation. However, it remains unclear whether the optical and radio variability are produced by the same active regions or even related magnetic regions.

\section{TOPOLOGY OF THE RADIO EMISSION REGION}

With a rotation period of $3.89 \mathrm{hr}$ and $v \sin i=45 \pm 5 \mathrm{~km}$ $\mathrm{s}^{-1}$, the minimum radius of $2 \mathrm{M} 1314+1320$ is inferred to be $9 \times 10^{9} \mathrm{~cm}\left(0.13 \mathrm{R}_{\odot}\right)$. The expected mass for an M7 dwarf Baraffe \& Chabrier (1996) is about $0.09 \mathrm{M}_{\odot}$, and the expected radius is therefore about $0.1 \mathrm{R}_{\odot}$ (Demory et al. 2009). Thus, the inferred radius is larger than predicted, especially if $i \ll 90^{\circ}$, and this may be indicative of the trend for larger radii than model predictions (by up to $\sim 50 \%$ ) for magnetically active ultracool dwarfs (Chabrier et al. 2007; López-Morales 2007; Jackson et al. 2009). Given that the minimum radius is already $40 \%$ larger than the model predictions, this suggests that that inclination is likely $\gtrsim 70^{\circ}$. However, since the binary companion is unresolved in $K$-band observations, the magnitude of the primary is difficult to estimate. $K$-band magnitude-mass relations indicate the mass may actually be higher $\left(0.1-0.17 \mathrm{M}_{\odot}\right)$ and the relatively low temperature may be a result of the inflated radius. This may resolve some of the discrepancy between the radius and $T_{\text {eff }}$.

The periodic radio emission, and especially the periodic helicity reversals at $4.86 \mathrm{GHz}$, can be consistently explained with a simple geometric model: a large-scale dipolar magnetic field mis-aligned relative to the rotation axis and with opposite polarity at each pole; see Figure 12. The inclination of the magnetic axis with respect to the observer leads to a larger projected surface area for one of the poles, and hence an increase in the total intensity when it rotates into view. At $4.86 \mathrm{GHz}$ we observe a helicity reversal when the other pole is in view. The fraction of circular polarization remains constant, but may actually be higher than observed if the inclination is such that both spots always remain partially visible. If this is the case, the opposite polarities of the two spots would cause some of the circular polarization to cancel out. The lack of obvious helicity reversals at $8.46 \mathrm{GHz}$ could be due 
to changes in the optical depth and the apparent size of the two magnetic poles as a function of frequency, particularly if higher frequencies are emitted near the magnetic foot-points. This is also supported by the smaller variability amplitude at $8.46 \mathrm{GHz}$.

The optical periodicity may be caused by cool spots spatially connected with the radio-emitting poles, or by unrelated spots, which are relatively common in M dwarfs. As noted above, spatial separation and differential rotation may explain the small difference in inferred optical and radio periods.

Morin et al. (2010) propose two distinct magnetic topologies of mid- to late-M dwarfs: large-scale, axisymmetric poloidal topology, and a complex, non-axisymmetric topology. Our geometric model indicates that 2M 1314+1320 has a topology more similar to the large-scale poloidal fields rather than those with complex, small-scale fields. The reason for these two populations remains unclear, but Morin et al. (2010) propose that objects may transition towards largerscale poloidal fields with age. We note, however, that the objects studied in Morin et al. (2010) have much slower rotation velocities (or correspondingly larger Rossby numbers) than $2 \mathrm{M} 1314+1320$.

\section{CONCLUSIONS}

2M 1314+1320 provides a unique window into magnetically-induced radio emission in ultracool dwarfs, coinciding with the spectral type at which the radio/X-ray correlation breaks down (Berger 2002, 2006). It has the most luminous stable radio emission over the widest frequency range $(1.4-22.5 \mathrm{GHz})$ to date, and furthermore exhibits clear sinusoidal periodicity, with the first example of a helicity reversal in the circularly polarized emission of an ultracool dwarf. Taken in conjunction, these properties point to emission from a simple dipolar field, mis-aligned relative to the stellar rotation axis. In the context of electron cyclotron maser emission, the magnetic field strength is required to be at least $8 \mathrm{kG}$. The inferred field structure is similar to the simple field topologies inferred from Zeeman Doppler imaging of some fully convective dwarfs (Morin et al. 2010), while the field strength in the case of ECM emission is larger than the typical average fields measured from Zeeman broadening of FeH lines (Reiners \& Basri 2007).

The stability of the radio emission for at least a decade in- dicates that the underlying dynamo is stable well beyond the convective turnover timescale for an M7 dwarf $(\sim 70 \mathrm{~d}$; Saar 2001). Moreover, a comparison to convective dynamo models and simulations supports predictions of dominant large-scale, low-multipole fields (Dobler et al. 2006; Browning 2008), particularly for fast rotators (Browning 2008), but is at odds with models that predict small-scale, high-multipole fields (Durney et al. 1993; Chabrier \& Küker 2006).

Future observations of 2M 1314+1320 will address several key questions. First, if the emission is due to the ECM process, we expect a cut-off in the radio emission at a frequency that corresponds to the maximal field strength, $\nu_{\max }=$ $2.8 \times 10^{6} B_{\max } \mathrm{Hz}$. Measurements of this possible cut-off frequency will therefore provide a crucial constraint on the magnetic field since the Zeeman techniques are only sensitive to the average surface field or its large-scale component. The increased sensitivity and bandwidth of the EVLA, particularly at frequencies of $\sim 20-40 \mathrm{GHz}$, is well-suited for this test. Second, simultaneous long-term monitoring of the radio and optical emission will test whether the variability has a similar period and phase. If identical, this will point to an origin in a related magnetic field structure, while a clear difference in period will be suggestive of differential rotation and an origin at different latitudes. Finally, long-term astrometric monitoring with the VLBA will be able to uncover a companion down to a few Jupiter masses (Forbrich \& Berger 2009). Thus, further studies of $2 \mathrm{M} 1314+1320$ are bound to provide new insights into the properties of fully convective dynamos and the release of magnetic energy in the coolest stars and brown dwarfs.

The authors would like to acknowledge assistance from Philip Nutzman, Zachory Berta, and Peter Challis. E.B. acknowledges support for this work from the National Science Foundation under Grant AST-1008361. J.I. gratefully acknowledges funding for the MEarth project from the David and Lucile Packard Fellowship for Science and Engineering (awarded to David Charbonneau), and support from the National Science Foundation under grant number AST-0807690. A.R. received research funding from the DFG as an Emmy Noether fellow (RE 1664/4-1). This paper includes data gathered with the 6.5 meter Magellan Telescopes located at Las Campanas Observatory, Chile.

\section{REFERENCES}

Bailer-Jones, C. A. L. 2002, A\&A, 389, 963

Baraffe, I., \& Chabrier, G. 1996, ApJ, 461, L51

Benz, A. O., \& Guedel, M., 285, 621

Berger, E. 2002, ApJ, 572, 503

Berger, E. 2006, ApJ, 648, 629

Berger, E., et al. 2001, Nature, 410, 338

Berger, E., et al. 2010, ApJ, 709, 332

Berger, E., et al. 2008, ApJ, 676, 1307

Berger, E., et al. 2009, ApJ, 695, 310

Berger, E., et al. 2005, ApJ, 627, 960

Browning, M. K. 2008, ApJ, 676, 1262

Chabrier, G., Gallardo, J., \& Baraffe, I. 2007, A\&A, 472, L17

Chabrier, G., \& Küker, M. 2006, A\&A, 446, 1027

Demory, B.-O., et al. 2009, A\&A, 505, 205

Dobler, W., Stix, M., \& Brandenburg, A. 2006, ApJ, 638, 336

Donati, J.-F., Forveille, T., Collier Cameron, A., Barnes, J. R., Delfosse, X., Jardine, M. M., \& Valenti, J. A. 2006, Science, 311, 633

Donati, J.-F., et al. 2008, MNRAS, 390, 545

Durney, B. R., De Young, D. S., \& Roxburgh, I. W., 145, 207

Forbrich, J., \& Berger, E. 2009, ApJ, 706, L205

Güdel, M. 2002, ARA\&A, 40, 217

Guedel, M., \& Benz, A. O. 1993, ApJ, 405, L63
Hallinan, G., Antonova, A., Doyle, J. G., Bourke, S., Brisken, W. F., \& Golden, A. 2006, ApJ, 653, 690

Hallinan, G., Antonova, A., Doyle, J. G., Bourke, S., Lane, C., \& Golden, A. 2008, ApJ, 684, 644

Hallinan, G., et al. 2007, ApJ, 663, L25

Irwin, J., Berta, Z. K., Burke, C. J., Charbonneau, D., Nutzman, P., West, A. A., \& Falco, E. E. 2011, ApJ, 727, 56

Irwin, J., Irwin, M., Aigrain, S., Hodgkin, S., Hebb, L., \& Moraux, E. 2007, MNRAS, 375, 1449

Jackson, R. J., Jeffries, R. D., \& Maxted, P. F. L. 2009, MNRAS, 399, L89

Law, N. M., Hodgkin, S. T., \& Mackay, C. D. 2006, MNRAS, 368, 1917

Lépine, S., \& Shara, M. M. 2005, AJ, 129, 1483

Lépine, S., Thorstensen, J. R., Shara, M. M., \& Rich, R. M. 2009, AJ, 137, 4109

Littlefair, S. P., Dhillon, V. S., Marsh, T. R., Shahbaz, T., Martín, E. L., \& Copperwheat, C. 2008, MNRAS, 391, L88

López-Morales, M. 2007, ApJ, 660, 732

McLean, M., \& Berger, E. 2011, ApJ, submitted

Mohanty, S., \& Basri, G. 2003, ApJ, 583, 451

Morin, J., et al. 2008, MNRAS, 390, 567

Morin, J., Donati, J.-F., Petit, P., Delfosse, X., Forveille, T., \& Jardine, M. M. 2010, MNRAS, 407, 2269

Nutzman, P., \& Charbonneau, D. 2008, PASP, 120, 317 
Osten, R. A., Hawley, S. L., Bastian, T. S., \& Reid, I. N. 2006, ApJ, 637, 518

Ravi, V., Hallinan, G., Hobbs, G., \& Champion, D. J. 2011, ApJ, 735, L2

Reiners, A. 2010, arXiv:1012.1183

Reiners, A., \& Basri, G. 2006, ApJ, 644, 497

Reiners, A., \& Basri, G. 2007, ApJ, 656, 1121

Reiners, A., \& Basri, G. 2008, ApJ, 684, 1390

Reiners, A., \& Basri, G. 2010, ApJ, 710, 924

Rest, A., et al. 2005, ApJ, 634, 1103
Rockenfeller, B., Bailer-Jones, C. A. L., \& Mundt, R. 2006, A\&A, 448, 1111 Saar, S. H. 2001, in Astronomical Society of the Pacific Conference Series, Vol. 223, 11th Cambridge Workshop on Cool Stars, Stellar Systems and the Sun, ed. R. J. Garcia Lopez, R. Rebolo, \& M. R. Zapaterio Osorio, 292 Treumann, R. A. 2006, A\&A Rev., 13, 229 
TABLE 1

VLA OBSERVATIONS OF 2M 1314+1320

\begin{tabular}{cccccc}
\hline \hline UT Date & Config. & $\begin{array}{c}\text { Duration } \\
(\mathrm{hr})\end{array}$ & $\begin{array}{c}\text { Frequency } \\
(\mathrm{GHz})\end{array}$ & $\begin{array}{c}F_{\nu} \\
(\mu \mathrm{Jy})\end{array}$ & $\begin{array}{c}F_{\nu, V^{a}} \\
(\mu \mathrm{Jy})\end{array}$ \\
\hline 2007 Jul 20.02 & $\mathrm{A}$ & 1 & 8.46 & $1156 \pm 27$ & $215 \pm 26$ \\
2007 Aug 10.00 & $\mathrm{A}$ & 1 & 8.46 & $1144 \pm 44$ & $227 \pm 43$ \\
2009 Mar 25.12 & $\mathrm{B}$ & 10 & 4.86 & $1099 \pm 18$ & $38 \pm 17$ \\
2009 Mar 25.12 & $\mathrm{B}$ & 10 & 8.46 & $1032 \pm 16$ & $117 \pm 16$ \\
2009 Mar 27.32 & $\mathrm{B}$ & 1 & 4.86 & $1063 \pm 39$ & $<84$ \\
2009 Mar 27.32 & $\mathrm{B}$ & 1 & 8.46 & $1052 \pm 42$ & $196 \pm 36$ \\
2009 May 10.42 & $\mathrm{B}$ & 1 & 8.46 & $942 \pm 30$ & $162 \pm 28$ \\
2009 May 11.37 & $\mathrm{B}$ & 1 & 1.43 & $890 \pm 58$ & $-218 \pm 6$ \\
2009 May 11.41 & $\mathrm{B}$ & 1 & 22.5 & $763 \pm 84$ & $<225$ \\
\hline
\end{tabular}

Note. $-{ }^{a}$ Circularly polarized flux density. 


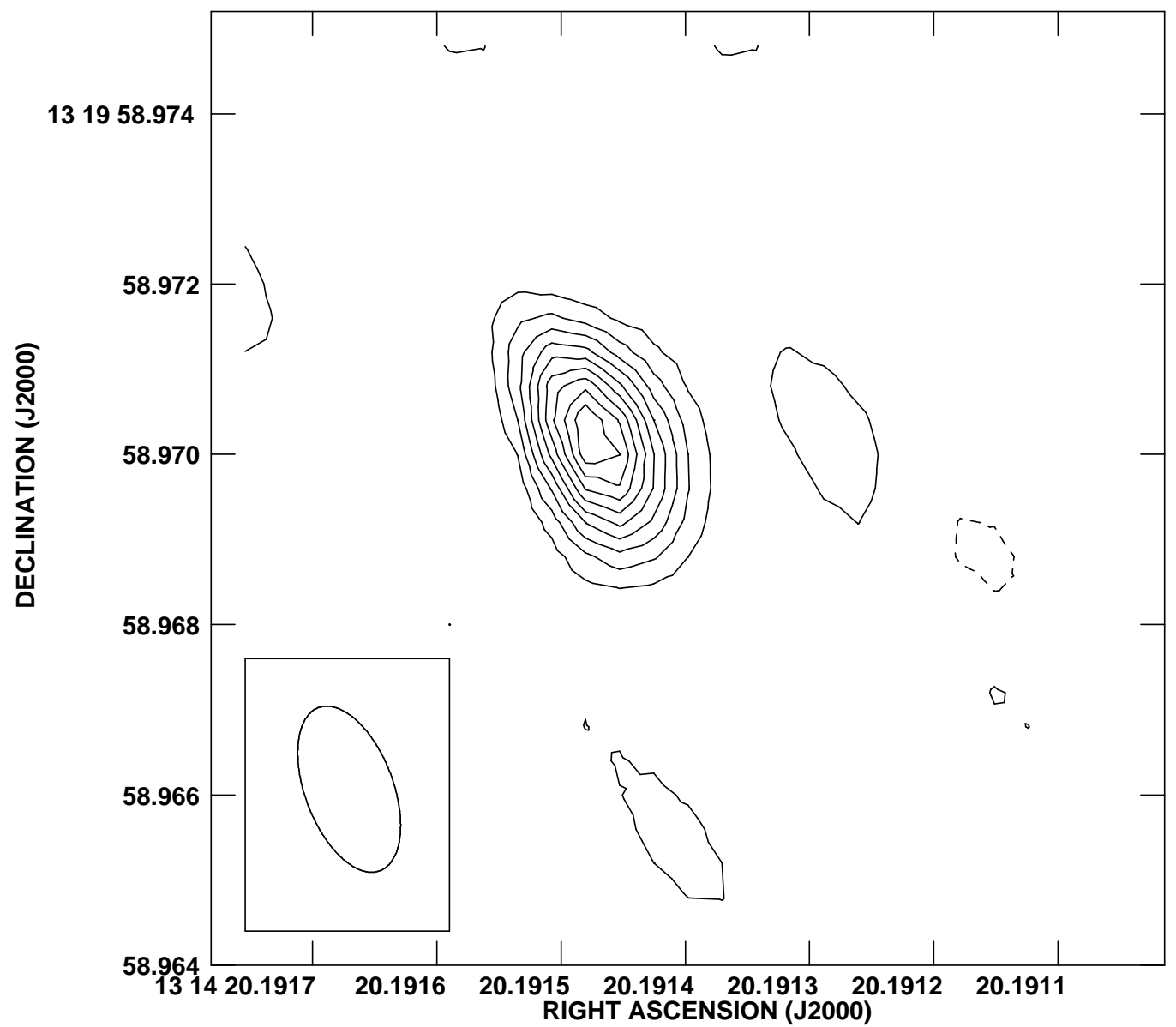

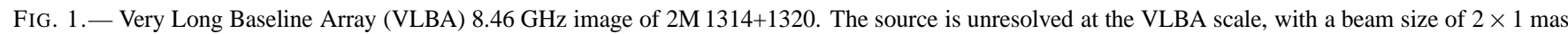
(bottom left). 

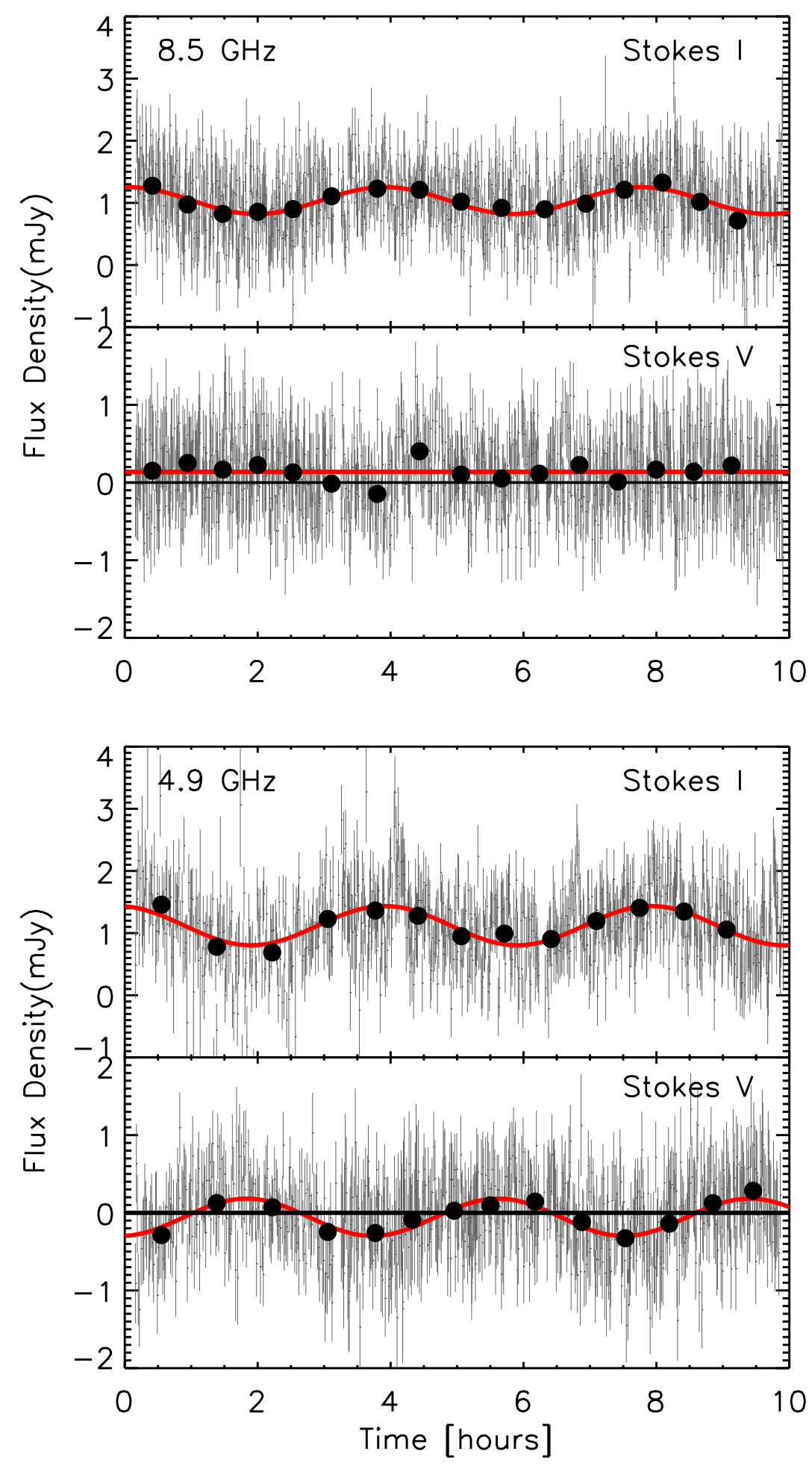

FIG. 2.- Radio light curves for the $10 \mathrm{hr}$ observation of $2 \mathrm{M} 1314+1320$ at $8.46 \mathrm{GHz}$ (top) and $4.86 \mathrm{GHz}$ (bottom). The top panel at each frequency shows the total intensity (Stokes I) while the bottom panels display the circularly polarized flux (Stokes V). The 30-s averaged data are shown in grey, with 25 -min averages shown as black circles. Sinusoidal periodicity is clearly visible at both frequencies. The best fit sinusoidal models are show in red, corresponding to a $22 \pm 3 \%$ variation with a $3.9 \pm 0.1 \mathrm{hr}$ period at $8.46 \mathrm{GHz}$, and $32 \pm 4 \%$ variation with a $4.0 \pm 0.1 \mathrm{hr}$ period at $4.86 \mathrm{GHz}$. The polarization light curve at $8.46 \mathrm{GHz}$ does not show significant periodic variation, while at $4.86 \mathrm{GHz}$ it alternates between right- and left-handed circular polarization over a best fit period of $3.8 \pm 0.4 \mathrm{hr}$ with an amplitude of $24 \pm 10 \%$. 


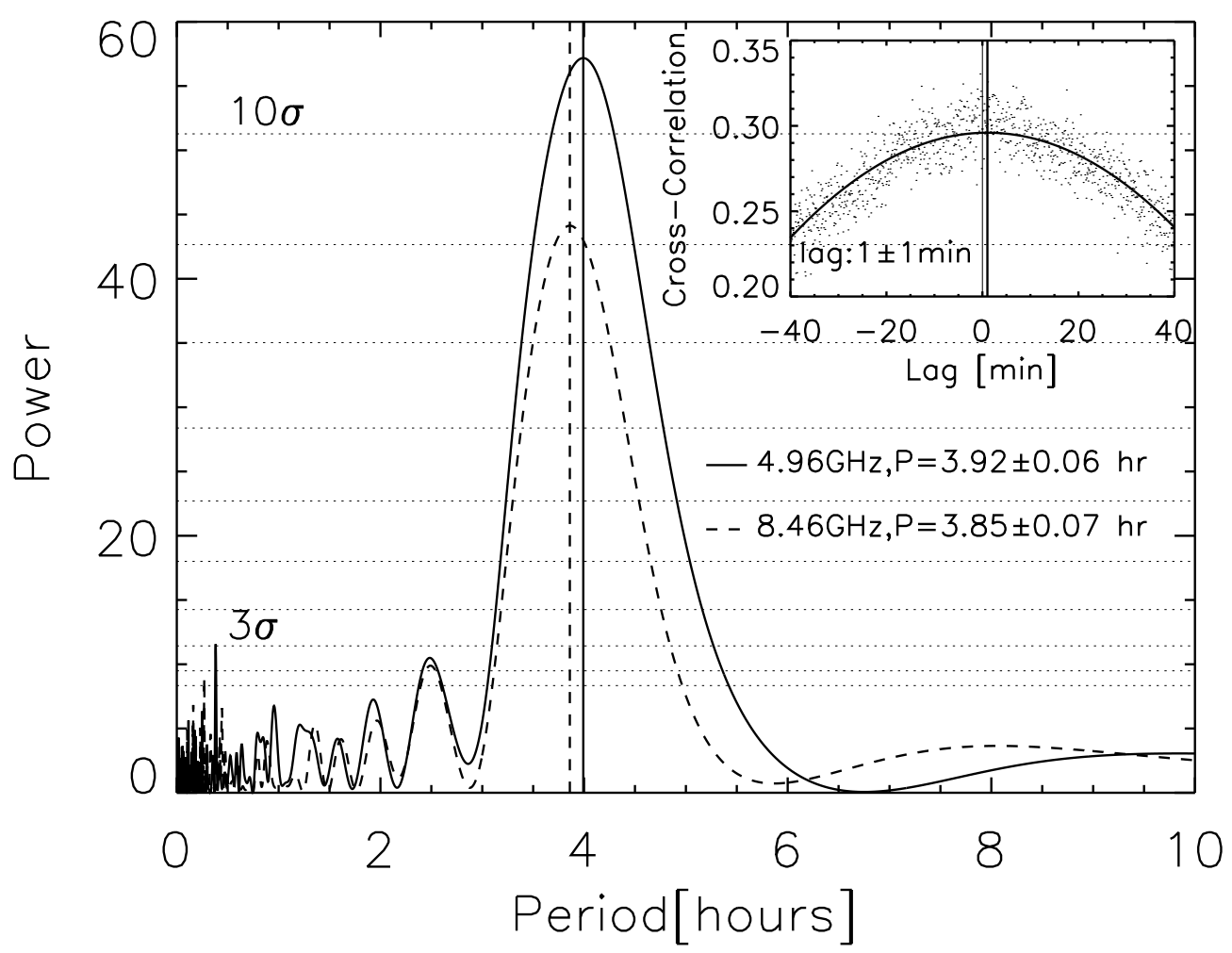

FIG. 3.- Lomb-Scargle periodograms for the total intensity data at $4.86 \mathrm{GHz}$ (solid line) and $8.46 \mathrm{GHz}$ (dashed line). A strong peak is seen at $3.92 \pm 0.06 \mathrm{hr}$ and $3.85 \pm 0.08 \mathrm{hr}$, respectively. Significance levels of $1-10 \sigma$ are plotted as horizontal lines. Also shown is a cross-correlation of the data from both frequencies (inset), with a peak at $1 \pm 1$ min indicating no discernible lag between the two light curves. 


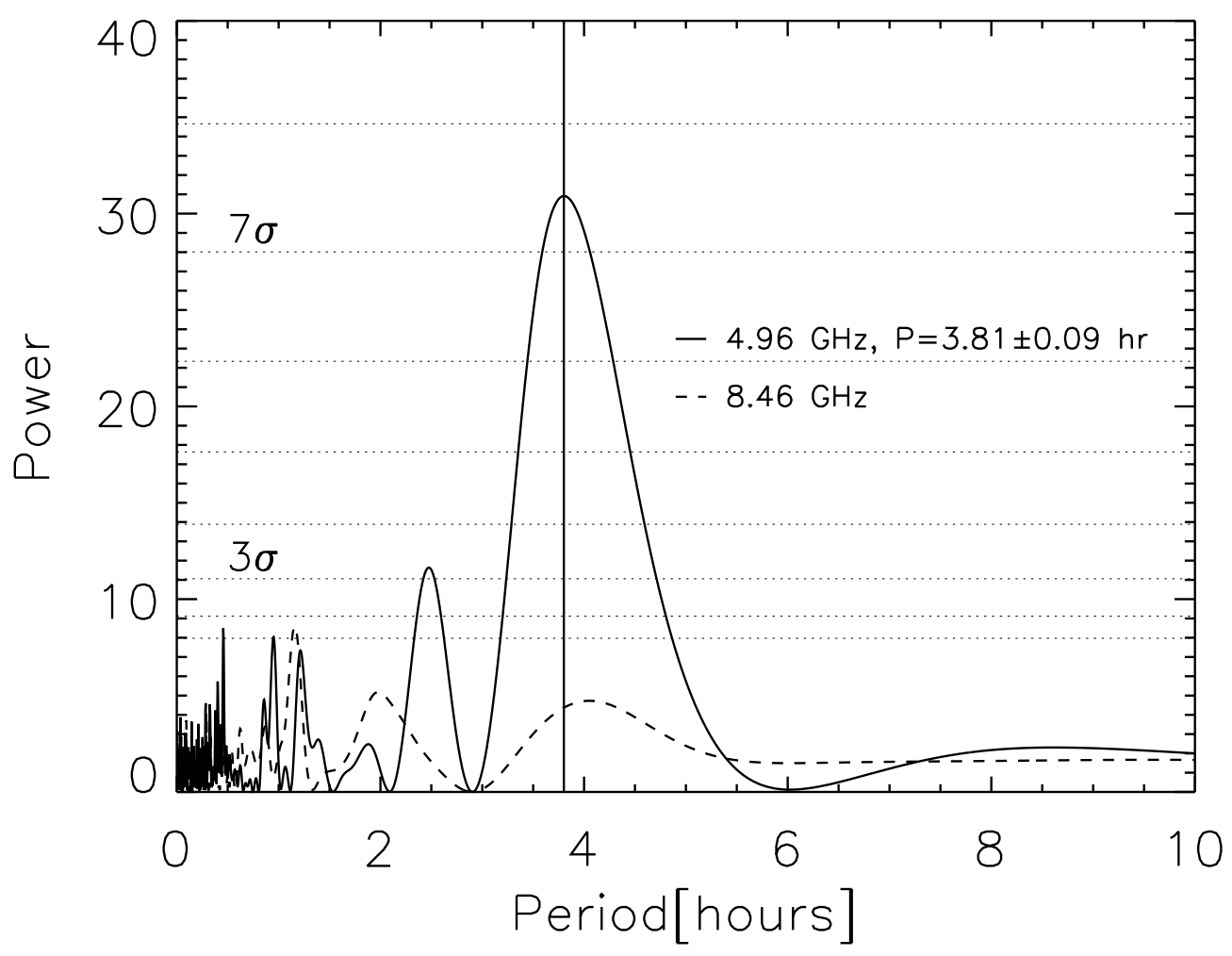

FIG. 4.- Same as Figure 3 but for the circular polarization light curves. A strong peak is seen at $3.81 \pm 0.09 \mathrm{hrs}$ in the $4.86 \mathrm{GHz}$ data, while no significant periodicity is evident at $8.46 \mathrm{GHz}$. Significance levels of $1-8 \sigma$ are plotted as horizontal lines. 


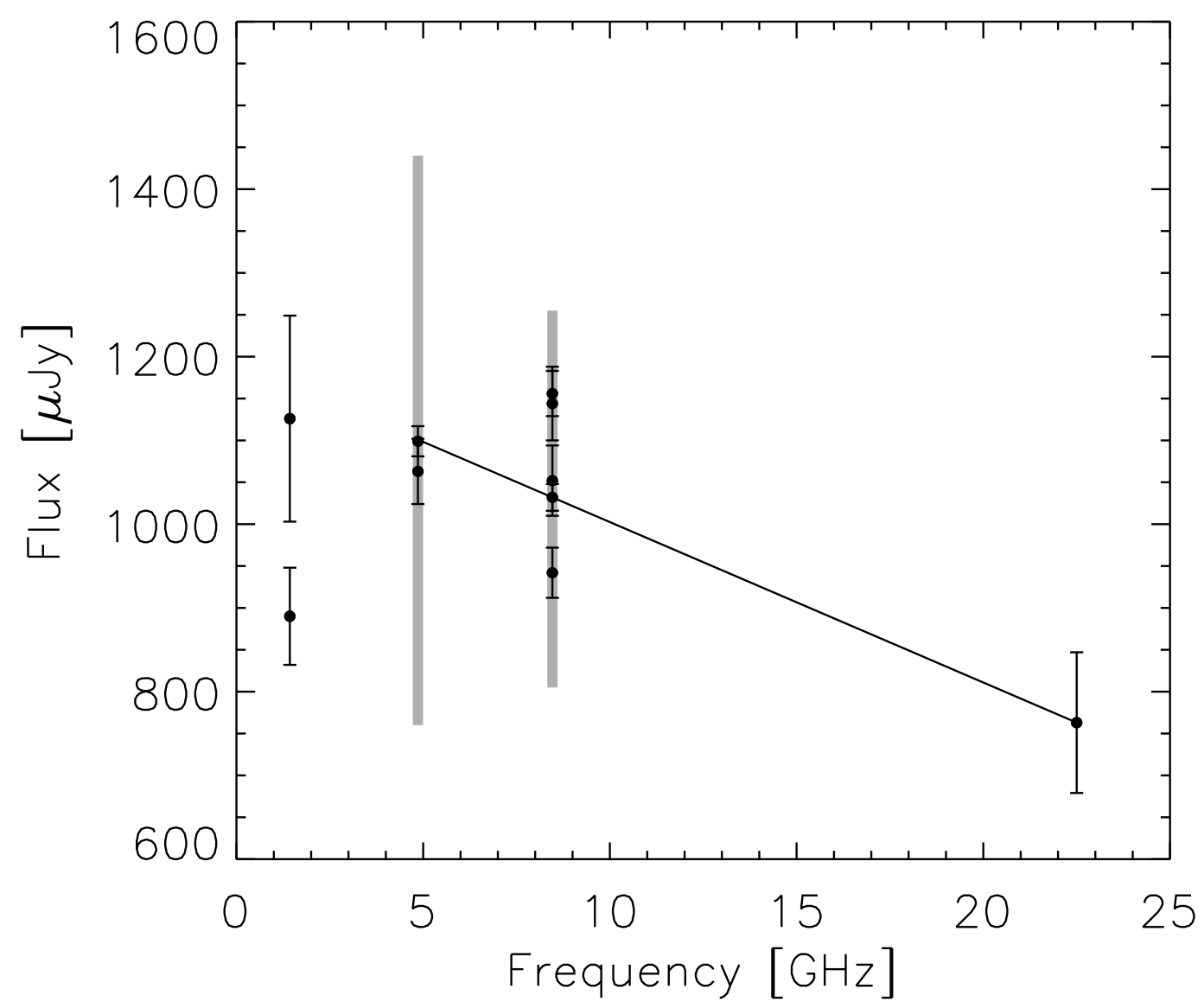

FIG. 5.- Radio spectral energy distribution of $2 \mathrm{M} 1314+1320$ from 1.4 to $22.5 \mathrm{GHz}$. Measurements from several short observations are shown in addition to the total flux density range detected in the 10 hour observations (vertical gray bars). The short observations at 1.4 and $22.5 \mathrm{GHz}$ provide no indication of the variability and may not represent the mean intensity at these frequencies. The spectrum is surprisingly flat and appears to peak around $5 \mathrm{GHz}$ with a spectral index of about -0.2 at higher frequencies. 


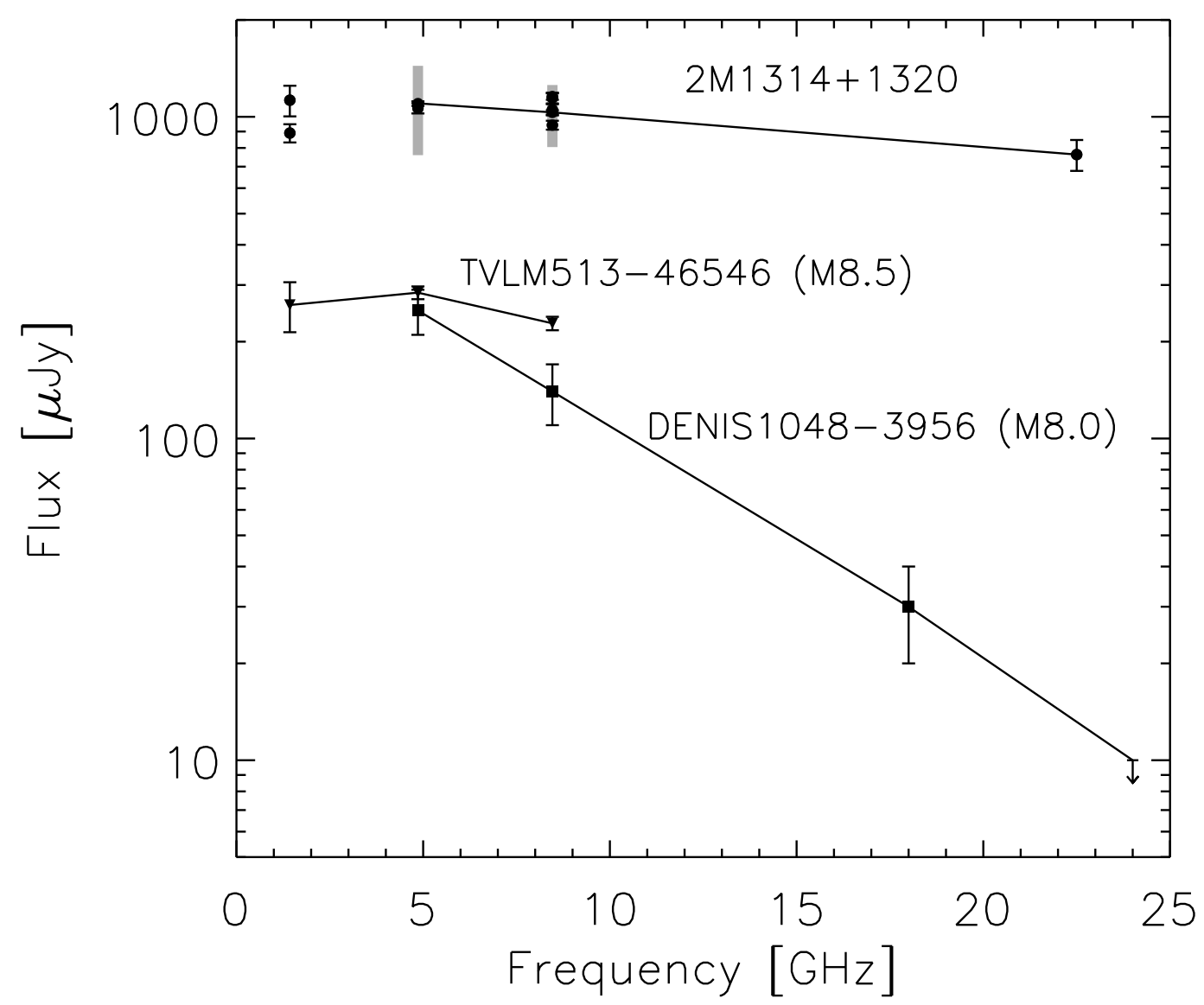

FIG. 6. - Comparison of the radio spectral energy distribution of 2M 1314+1320 with other ultracool dwarfs: TVLM 513-46546 (M8.5; Osten et al. 2006) and DENIS 1048-3956 (M8; (Ravi et al. 2011). The SED of 2M 1314+1320 is significantly flatter than that of DENIS 1048-3956 


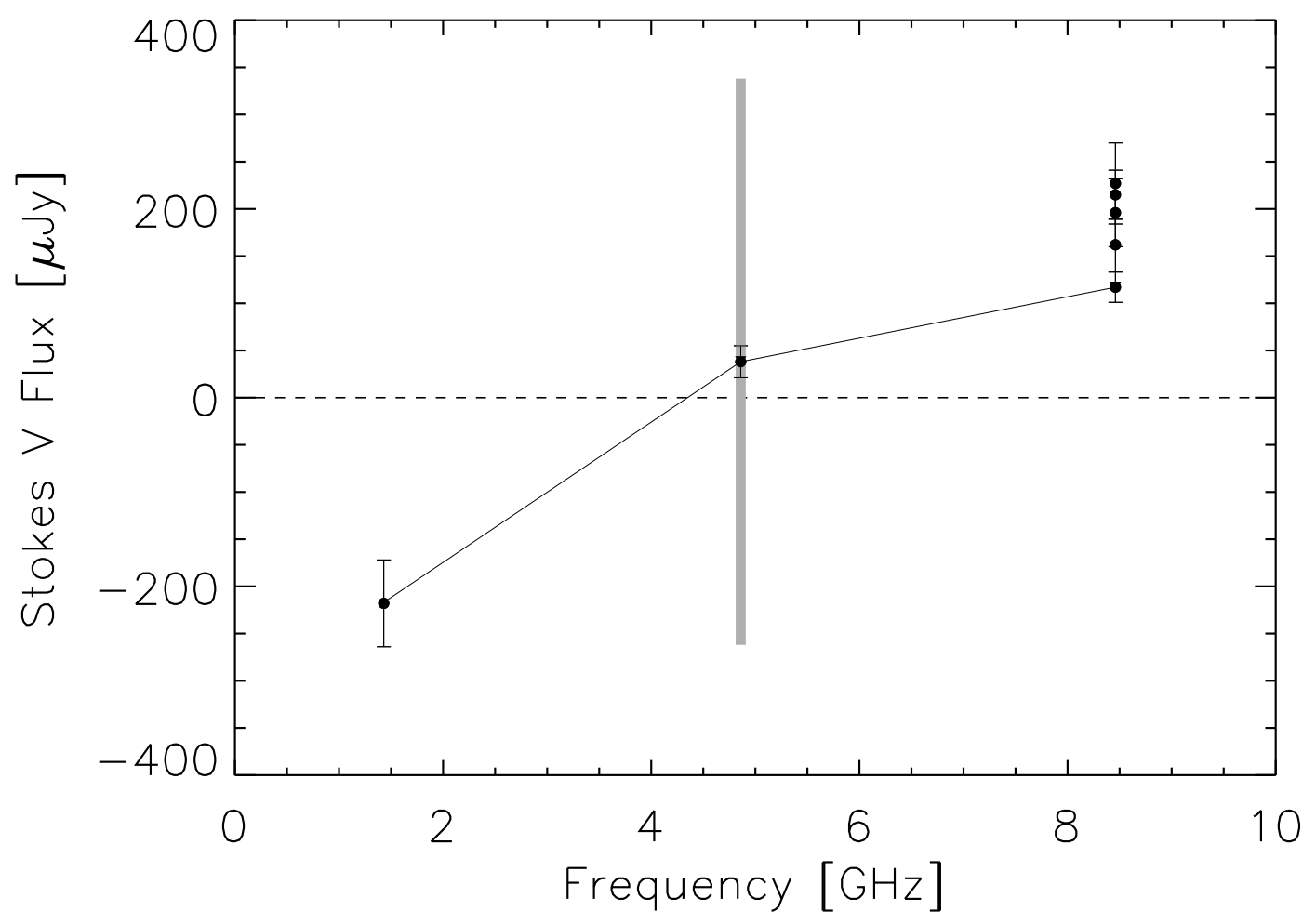

FIG. 7.- Same as Figure 5 but for the circularly polarized flux. It is unclear if the single 1-hr observation at $1.4 \mathrm{GHz}$ represents the true mean polarization or if the object varies from right- to left-handed circular polarization as it does at $4.86 \mathrm{GHz}$. The polarization is consistently positive at $8.46 \mathrm{GHz}$, but is lowest during the 10 -hr observation. 


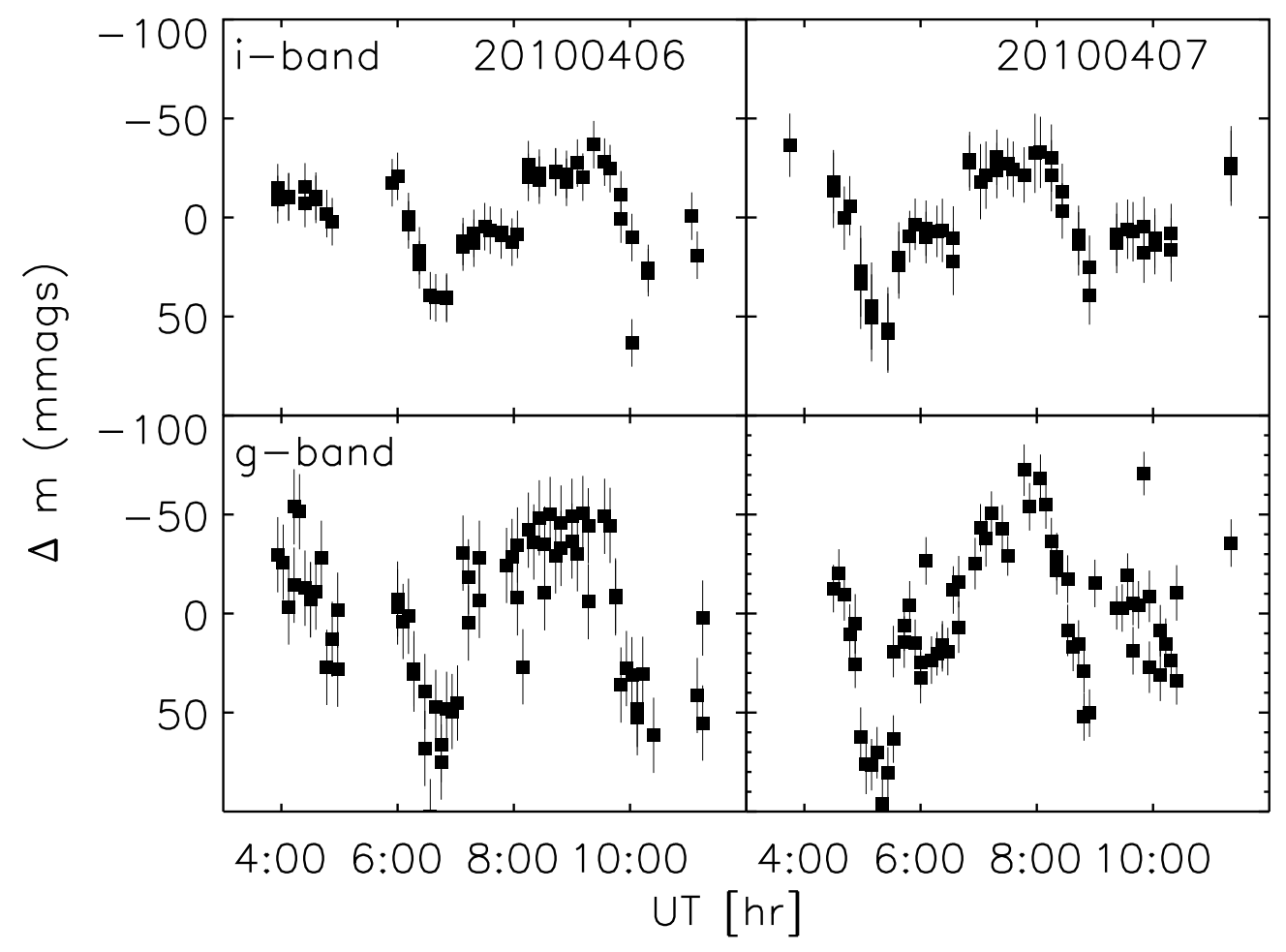

FIG. 8.- Optical $g$ - and $i$-band light curves from two nights of observations at the FLWO 1.2-m. A period of about $4 \mathrm{hr}$ is evident. 


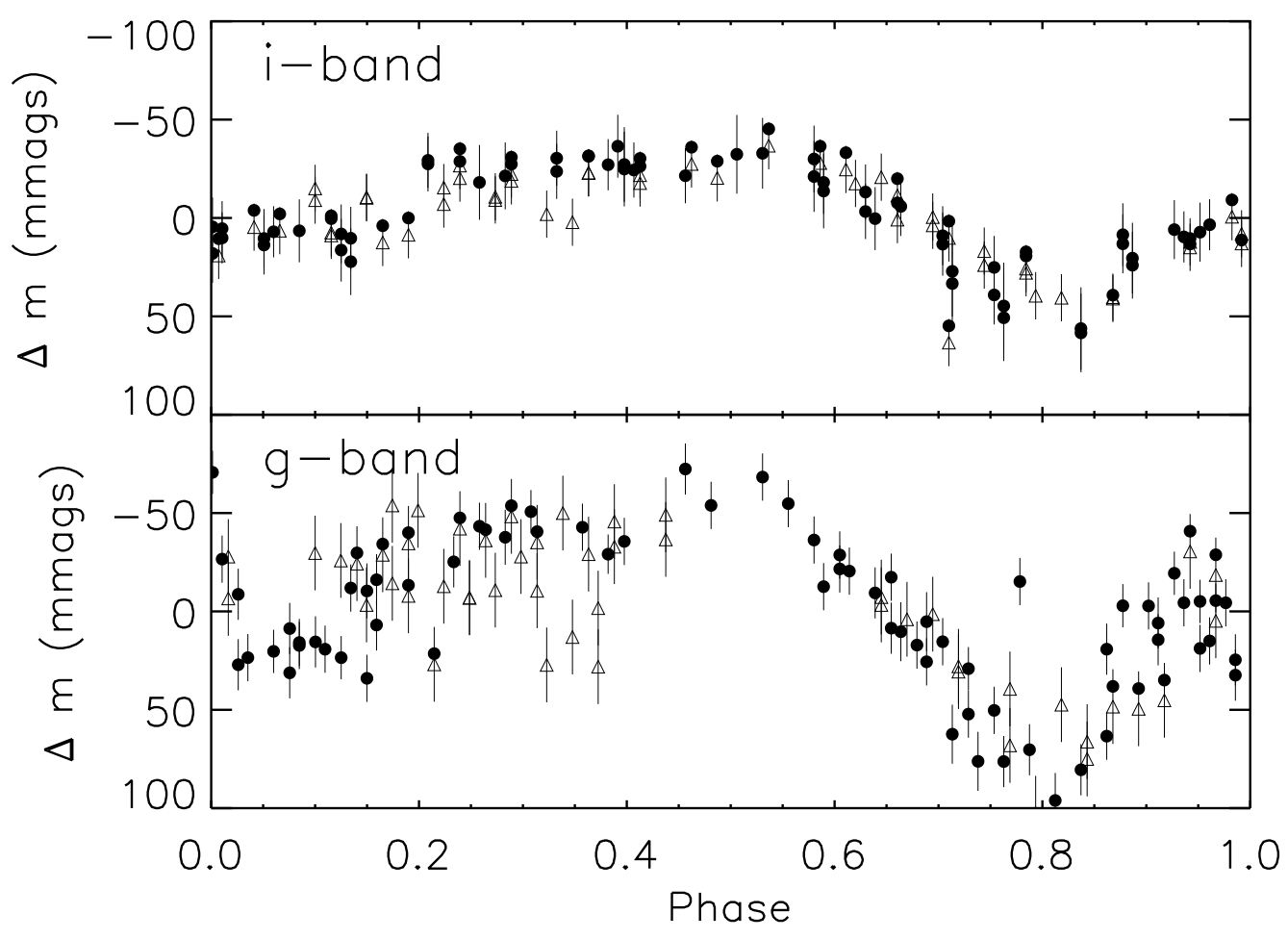

FIG. 9.- Phased light curves of the data in Figure 8 folded with a period of $3.78 \mathrm{hr}$. Data from the two consecutive nights are plotted as triangles and circles, respectively. The shape of the phased light curve appears more complex than a simple sinusoid. 


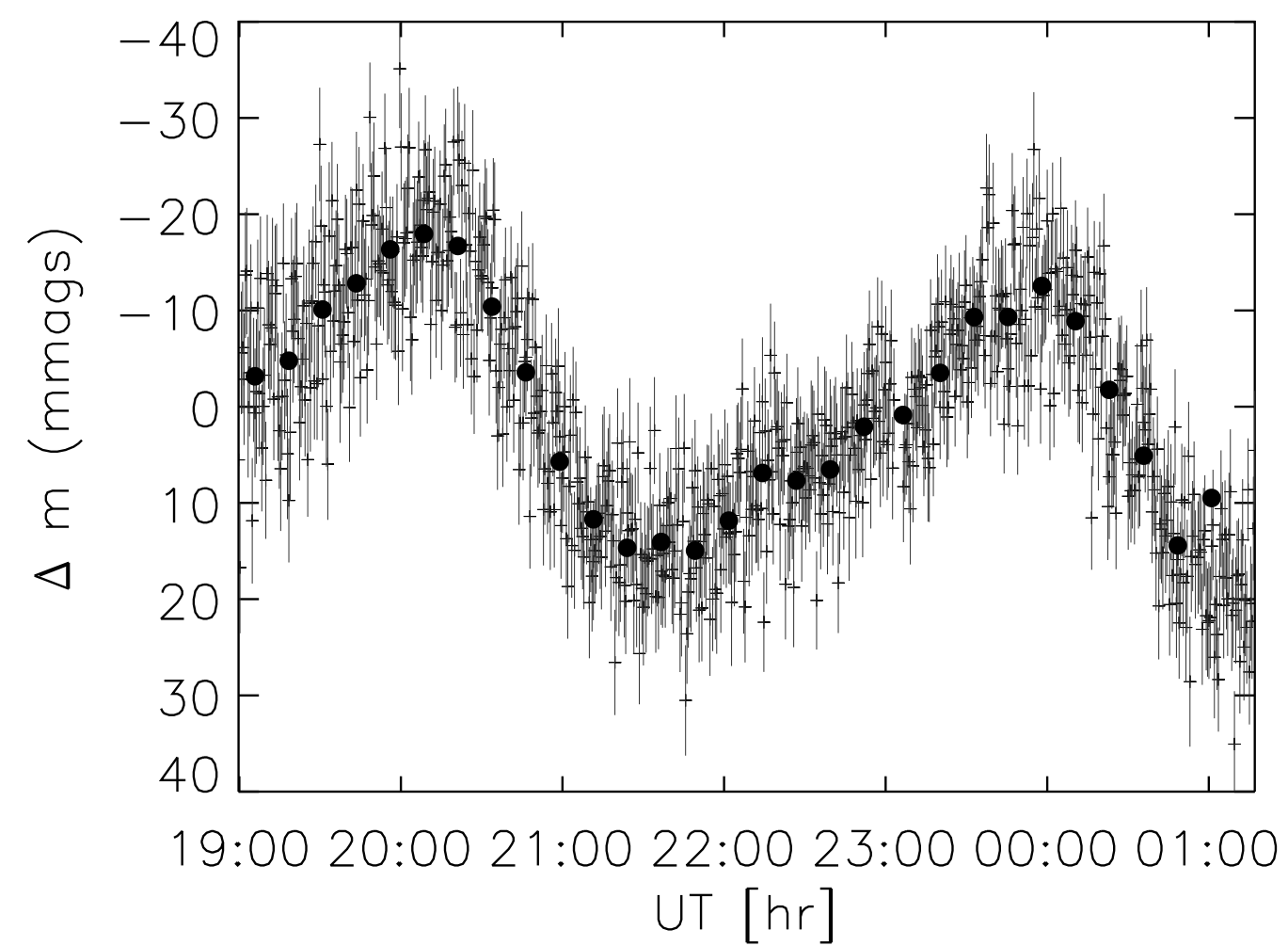

FIG. 10.- A subset of high cadence photometric observations from the MEarth telescopes. A period of $3.78 \mathrm{hr}$ is evident with an asymmetric light curve. 


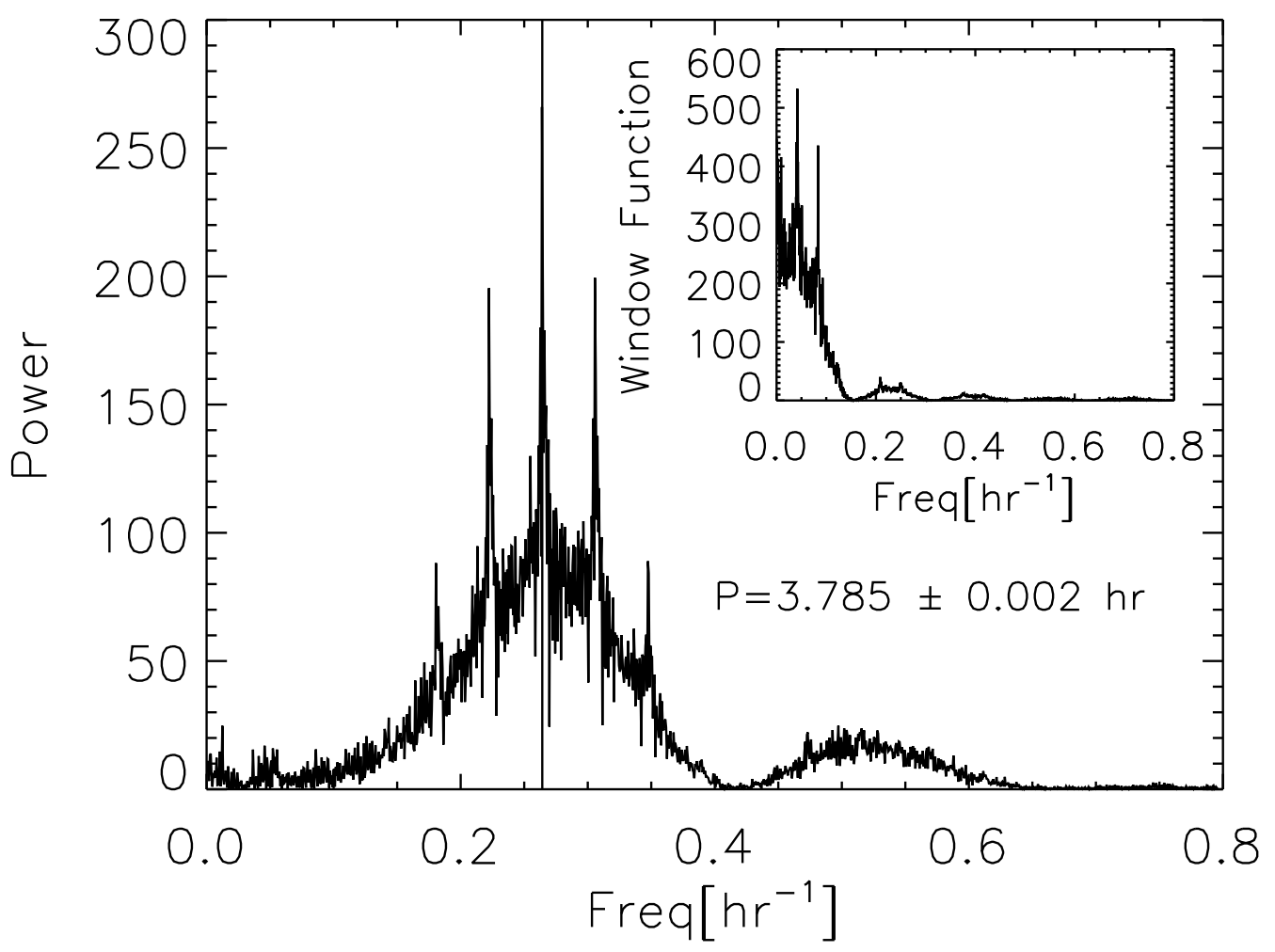

FIG. 11. - Lomb-Scargle periodogram of optical observations from the MEarth telescopes. A clear peak is seen at a period of $P=3.785 \pm 0.002$ hr. Aliased or harmonic peaks are also visible due to the window function produced by the uneven sampling of the light curve (inset). 

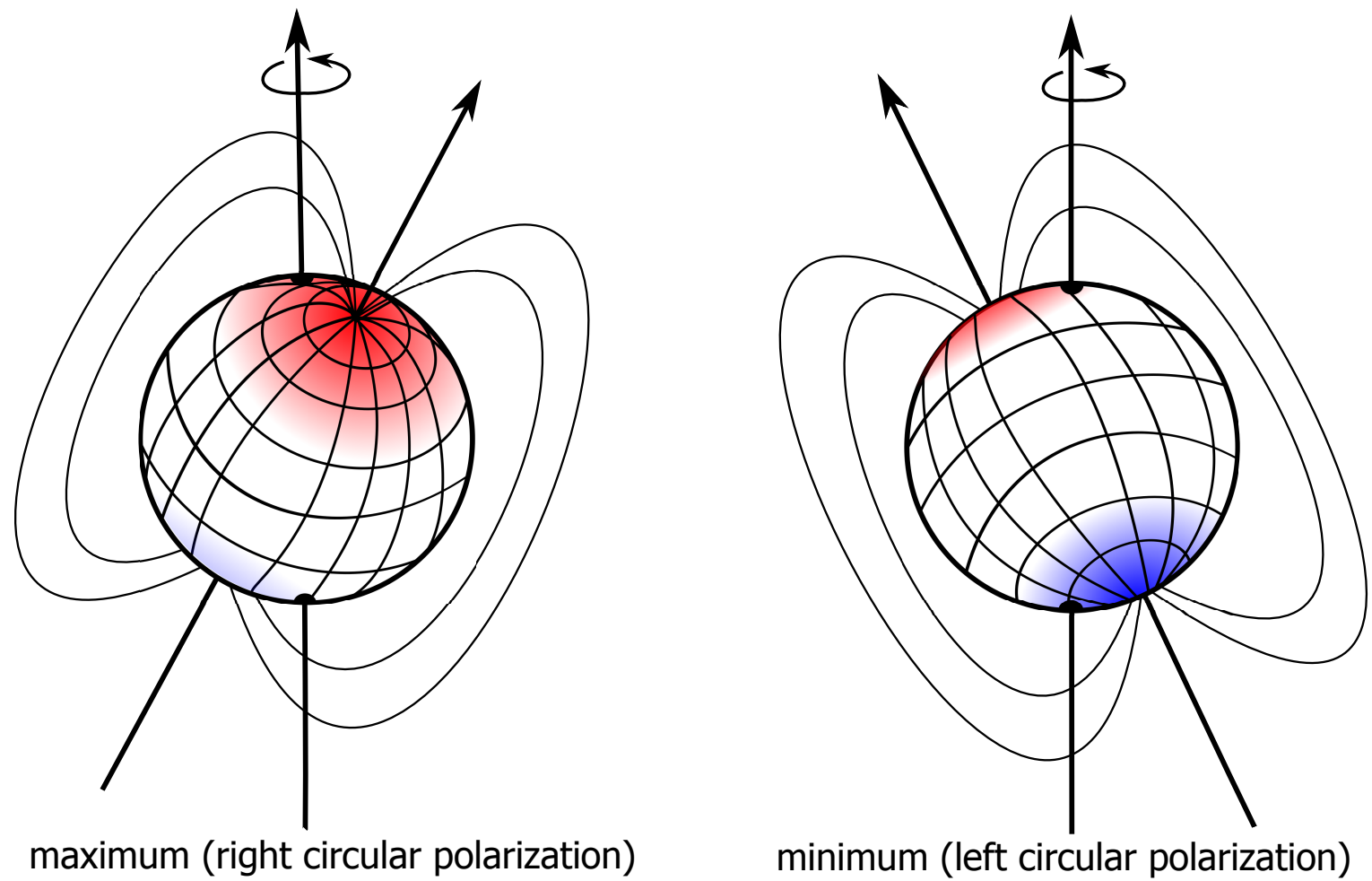

FIG. 12.- Simple geometric model of a mis-aligned dipolar magnetic field that can explain the various properties of the observed radio emission. The radio emission arises from two large magnetic hot spots with opposite polarities, located at opposite poles. The inclination with respect to the observer leads to a larger projected area from one of the poles (red) and hence a peak in the total intensity when it rotates into view. Helicity reversals are observed when the second pole (blue) is visible. 